\title{
Aspectos relevantes del tamiz neonatal para hiperplasia suprarrenal congénita
}

\section{Relevant aspects of the congenital adrenal hyperplasia newborn screening}

\author{
Mirna Angélica Hinojosa-Trejo, ${ }^{1}$ Sletza Lissete Arguinzoniz-Valenzuela, ${ }^{2}$ Luz del Alba Herrera-Pérez, ${ }^{3}$ Guillermo \\ Caamal-Parra, ${ }^{3}$ Isabel Ibarra-González, ${ }^{4}$ Marcela Vela-Amieva, ${ }^{5}$ Lidia Elizabeth Bolaños-Córdova, ${ }^{1}$ Iñaki Gon- \\ zález-Baqué, ${ }^{2}$ Erika Paola García-Flores ${ }^{1}$
}

\begin{abstract}
Resumen
INTRODUCCIÓN: El beneficio del tamiz neonatal de la hiperplasia suprarrenal congénita está demostrado y la determinación de la $17 \alpha \mathrm{OHP}(17-\alpha \mathrm{OHP})$ en sangre es el biomarcador más utilizado. En México no se conoce bien la prevalencia de esta enfermedad diagnosticada mediante tamiz neonatal.

OBJETIVO: Dar a conocer la prevalencia de hiperplasia suprarrenal congénita al nacimiento y describir los valores de $17 \alpha \mathrm{OHP}$ en recién nacidos mexicanos según el sexo, edad gestacional y edad de vida extrauterina al momento de la toma de la muestra, peso al nacimiento y condición de salud.

MATERIALES Y MÉTODOS: Estudio retrospectivo y longitudinal efectuado en recién nacidos entre el 1 de enero de 2017 y 31 de mayo de 2018 en unidades médicas de la Secretaría de Salud de México. En todos los sujetos de estudio se efectuó la determinación cuantitativa de $17 \alpha \mathrm{OHP}$ mediante fluoroinmunoensayo en sangre seca en papel filtro. Todas las muestras de recién nacidos con valor de $17 \alpha \mathrm{OHP}$ mayor de 20 $\mathrm{nmol} / \mathrm{L}$ se notificaron inmediatamente para su localización y efectuarles las pruebas confirmatorias.

RESULTADOS: La prevalencia al nacimiento de hiperplasia suprarrenal congénita obtenida en este trabajo fue de 12.6 por cada 100,000 recién nacidos, es decir, 1:7920 recién nacidos tamizados. Las concentraciones de $17 \alpha \mathrm{OHP}$ en sangre en papel filtro de los recién nacidos tuvieron diferencias significativas según el peso, edad gestacional y el estado de salud. La proporción de rellamados fue de $0.14 \%$. Un hallazgo de este estudio es el elevado número de recién nacidos sospechosos que fallecieron sin poder ser confirmados (106 muertos de 1820 sospechosos, 5.82\%).

CONCLUSIÓN: La prevalencia al nacimiento de hiperplasia suprarrenal congénita en este trabajo fue de 12.6 por cada 100,000 (1:7920) recién nacidos tamizados en la Secretaría de Salud de México. Para disminuir el número de casos sospechosos no localizados es necesario reforzar el componente postanalítico del tamiz neonatal.

PALABRAS CLAVE: Hiperplasia suprarrenal congénita; deficiencia de 21-hidroxilasa; $17 \alpha$-hidroxiprogesterona; tamiz neonatal.
\end{abstract}

\section{Abstract}

INTRODUCTION: The benefit of the newborn screening for congenital adrenal hyperplasia has been demonstrated and the determination of 17-ahydroxyprogesterone $(17 \alpha \mathrm{OHP})$ in blood is the frequent used biomarker. In Mexico the prevalence of this disease determined by newborn screening is not well known.

OBJECTIVE: To present the congenital adrenal hyperplasia birth prevalence obtained through newborn screening and to describe $17 \alpha \mathrm{OHP}$ blood concentrations in Mexican newborns according to sex, gestational age and extrauterine life age at the time of sampling, birth weight and health condition.
${ }^{1}$ Centro Nacional de Equidad de Género V Salud Reproductiva, Secretaría de Salud, México.

2 Servicio de Endocrinología, Instituto Nacional de Pediatría, Secretaría de Salud,

México

TamizMas Screening Center de Químicos Maldonado, Yucatán, México. 4 Unidad de Genética de la Nutrición, Instituto de Investigaciones Biomédicas, UNAM, México.

${ }^{5}$ Laboratorio de Errores Innatos del Metabolismo y Tamiz, Instituto Nacional de Pediatría, Secretaría de Salud, México.

Correspondencia

Erika Paola García Flores

erika.garcia@salud.gob.mx

\section{Este artículo debe citarse como} Hinojosa Trejo MA, Arguinzoniz Valenzuela SL,Herrera Pérez LA, Caamal Parra G, IbarraGonzález I, Vela Amieva M, Bolaños Córdova LE, González Baqué I, García Flores EP. Aspectos relevantes del tamiz neonatal para hiperplasia suprarrenal congénita.Acta Pediatr Mex. 2018;SI(39):14S-24-S. 
MATERIAL AND METHODS: Quantitative determination of $17 \alpha \mathrm{OHP}$ by immunoflourometric assay in dry blood on filter paper. All those newborns whose samples had a $17 \alpha \mathrm{OHP}$ blood concentration value $>20 \mathrm{nmol} / \mathrm{L}$, were immediately notified for their localization and confirmatory tests.

RESULTS: The birth prevalence of congenital adrenal hyperplasia obtained in this work was 12.6 per 100,000 , ie $1: 7,920$ screened newborns. The $17 \alpha \mathrm{OHP}$ blood concentrations had significant differences according to weight, gestational age and health status. The recall rate was $0.14 \%$. A finding of this study is the high number of suspicious newborns who died without being able to be confirmed (106 dead/1,820 suspects, $5.82 \%$ ).

CONCLUSION: The congenital adrenal hyperplasia birth prevalence was 12.6 per $100.000(1: 7,920)$ screened newborns. It is necessary to reinforce the newborn screening postanalytical component to reduce the number of non-localized and unconfirmed cases.

KEYWORDS: Congenital adrenal hyperplasia, 21-hydroxylase deficiency, 17 $\alpha$-hydroxyprogesterone, newborn screening.

\section{INTRODUCCIÓN}

La insuficiencia suprarrenal es la incapacidad en la formación o síntesis de cortisol. En 1865, Luigi De Crecchio, un patólogo italiano, reportó el primer caso conocido de hiperplasia suprarrenal congénita, al describir un individuo con anatomía externa masculina, interna femenina y glándulas suprarrenales de gran tamaño. ${ }^{1}$ Casi un siglo después, con el uso de la cortisona, se introdujo el tratamiento para síndrome adrenogenital. ${ }^{2}$ La hiperplasia suprarrenal congénita engloba un grupo de siete enfermedades monogénicas autosómicas recesivas causadas por mutaciones en los genes que codifican las vías enzimáticas de la biosíntesis de cortisol: 21-hidroxilasa $(21 \mathrm{OH}), 11 \beta$-hidroxilasa $(11 \beta \mathrm{OH})$, $17 \alpha$-hidroxilasa $(17 \alpha \mathrm{OH}$, también conocida como 17, 20-liasa), 3 $\beta$-hidroxiesteroide deshidrogenasa tipo 2 ( $3 \beta \mathrm{HSD} 2)$, proteína reguladora de la esteroidogénesis aguda (StAR, por sus siglas en inglés), enzima de escisión de la cadena lateral del colesterol P450 (SCC) y oxidorreductasa P450 (POR). ${ }^{2}$ El defecto común de todos estos trastornos es la disfunción de la síntesis de cortisol, que provoca hipersecreción de hormona liberadora de corticotropina $(\mathrm{CRH}$, por sus siglas en inglés) y hormona adrenocorticotropa (ACTH, por sus siglas en inglés) y consecuentemente hiperplasia de las glándulas suprarrenales. ${ }^{3}$

La hiperplasia suprarrenal congénita implica múltiples desequilibrios hormonales que pueden manifestarse con fenotipos clínicos y bioquímicos muy diferentes, con o sin trastornos de la producción de mineralocorticoides y esteroides sexuales. Se han descrito formas severas (clásicas) y leves (no clásicas) según el grado de afección de la enzima involucrada. Las formas clásicas, con déficit enzimático absoluto se manifiestan en la etapa neonatal; las formas no clásicas con deficiencias enzimáticas parciales casi siempre son de manifestación tardía (infancia, adolescencia o adultez), o incluso permanecen asintomáticas (formas crípticas). 2,4

\section{Epidemiología}

La hiperplasia suprarrenal congénita es la causa más frecuente de alteraciones en la diferenciación sexual. Más de 95\% de los casos de hiperplasia suprarrenal congénita son producidos por una deficiencia de $21 \mathrm{OH}$, caracterizada por menor producción de cortisol y aldosterona 
y exceso de andrógenos. Se estima que la incidencia de hiperplasia suprarrenal congénita clásica varía entre 1:10,000 a 1:20,000 recién nacidos y la forma perdedora de sal tiene una incidencia de 1:13,000 y ocurre en $75 \%$ de los casos de hiperplasia suprarrenal congénita. La frecuencia de la forma virilizante simple es cerca de 1:40,000 recién nacidos y sucede en $25 \%$ de los casos. ${ }^{5}$ Existe una gran variación en la frecuencia de esta enfermedad dependiendo del área geográfica y de la etnia estudiada; las poblaciones aisladas, como los esquimales Yupik de Alaska (1:280 recién nacidos vivos) y los habitantes de la isla francesa de la Reunión $(1: 2,100$ recién nacidos vivos) son quienes tienen mayor prevalencia. En Arabia Saudita (1:5,000 recién nacidos vivos), Brasil $(1: 7,500$ recién nacidos vivos) y Filipinas (1:7,000 recién nacidos vivos) también es frecuente. En cambio, la frecuencia es baja en Nueva Zelanda (1:21,270 recién nacidos vivos). En Estados Unidos la incidencia es menor en la población de origen africano (1:42,000 recién nacidos vivos) que en la población blanca (1:15,000 recién nacidos vivos). ${ }^{6}$ En México, el IMSS a nivel nacional reporta una incidencia de $1: 8,743 .^{7}$

\section{Patogénesis y manifestaciones clínicas}

Todos los tipos de hiperplasia suprarrenal congénita son monogénicos y la mayoría de los pacientes son heterocigotos compuestos (65-75\%). Hay una buena correlación genotipo-fenotipo. Las manifestaciones clínicas dependen de la actividad enzimática residual. ${ }^{2,8}$ Las manifestaciones clínicas de la hiperplasia suprarrenal congénita dependen de la enzima deficiente y severidad del bloqueo. ${ }^{2}$

Los programas de tamiz neonatal tienen como objetivo la identificación presintomática y el tratamiento temprano de los defectos metabólicos congénitos tratables, para reducir la morbilidad y mortalidad y las posibles discapacidades asociadas con esas enfermedades. ${ }^{6}$ El beneficio de la detección temprana de la hiperplasia suprarrenal congénita lo han demostrado diversos autores; ${ }^{9,10,11}$ sin embargo, el tamiz neonatal para esta enfermedad es complejo y tiene importantes dificultades, ${ }^{12}$ entre ellas la baja especificidad de los métodos analíticos inmunológicos que cuantifican la $17 \alpha \mathrm{OHP}$ y que pueden tener una reacción cruzada con los esteroides. ${ }^{13}$ Además, la $17 \alpha \mathrm{OHP}$ puede elevarse por el estrés secundario a cualquier enfermedad, sobre todo en los recién nacidos prematuros, lo que contribuye a un alto número de notificaciones de casos sospechosos, también conocidos como rellamados, que generalmente terminan siendo falsos positivos. ${ }^{12,14,15}$ Conocer los valores en sangre de la $17 \alpha \mathrm{OHP}$ en la población neonatal y su relación con los factores que la afectan es indispensable para hacer una adecuada interpretación del resultado del tamiz neonatal. En México, el Centro Nacional de Equidad de Género y Salud Reproductiva (CNEGSR) es la unidad de la Secretaría de Salud responsable de las políticas nacionales de los programas de Salud Materna y Perinatal, entre los que se incluye el tamiz metabólico neonatal. El tamiz neonatal para hiperplasia suprarrenal congénita se realiza a nivel nacional desde 2011, de conformidad con lo establecido en el artículo 61 de la Ley General de Salud y con la normatividad oficial vigente. ${ }^{16,17,18}$

El objetivo de este estudio fue: dar a conocer la prevalencia de hiperplasia suprarrenal congénita al nacimiento y describir los valores de $17 \alpha \mathrm{OHP}$ en recién nacidos mexicanos según el sexo, edad gestacional y edad de vida extrauterina al momento de la toma de la muestra, peso al nacimiento y condición de salud.

\section{MATERIALES Y MÉTODOS}

Estudio retrospectivo y longitudinal efectuado del 1 de enero de 2017 al 31 de mayo del 2018 en recién nacidos atendidos en unidades 
médicas de la Secretaría de Salud de México. En todos los sujetos de estudio se efectuó la determinación cuantitativa de $17 \alpha \mathrm{OHP}$ mediante fluoroinmunoensayo en sangre seca en papel filtro. Todas las muestras de recién nacidos con valor de $17 \alpha \mathrm{OHP}$ mayor de $20 \mathrm{nmol} / \mathrm{L}$ se notificaron inmediatamente para su localización y efectuarles las pruebas confirmatorias.

Para la toma de muestra solo se solicitó consentimiento informado verbal a los padres o tutores del recién nacido. ${ }^{16,17,18}$ Del total de recién nacidos tamizados, 6,605 (0.52\%) muestras se consideraron inadecuadas para el procesamiento bioquímico, ya sea por ser insuficientes las gotas de sangre, por tener algún tipo de contaminación visible, halos de suero o sobreposición de gotas de sangre.

\section{Datos demográficos}

La fecha de nacimiento, el peso al nacimiento, la edad al momento de la toma de la muestra, la existencia o no de prematuridad (menos de 37 semanas de gestación) y la condición de salud (sano o enfermo), se obtuvieron de la ficha de identificación de cada recién nacido y se consignaron en el reporte de datos del laboratorio.

\section{Métodos analíticos}

Cuantificación de $17 \alpha O H P$. La determinación cuantitativa de $17 \alpha \mathrm{OHP}$ humana se realizó en las gotas de sangre seca recolectadas en papel filtro mediante fluoroinmunoensayo a tiempo resuelto utilizando estuches de reactivos comerciales (PerkinElmer-Wallac Oy, Mustionkatu 6, FI-20750 Turku, Finlandia). Se utilizó el instrumento Genetic Screening Processor ${ }^{\circledR}$ (GSP $^{\circledR}$ PerkinElmer), siguiendo las instrucciones del fabricante y empleando seis calibradores por duplicado y tres controles de concentraciones baja, media y alta en cada placa de 96 pocillos. Cada una de las muestras de sangre en papel filtro se perforó mediante instrumentos automatizados (Panthera-puncher-9, PerkinElmer ${ }^{\circledR}$ ) con los que se obtuvo un círculo estandarizado de $3.2 \mathrm{~mm}$ para proceder a la determinación bioquímica. Los valores de $17 \alpha \mathrm{OHP}$ se expresaron en $\mathrm{nmol} / \mathrm{L}$ de sangre (factor de conversión: 1 $\mathrm{nmol} / \mathrm{L}=0.33 \mathrm{ng} / \mathrm{mL}$ ).

Se siguió el algoritmo establecido por el CNEGSR en el que todas las muestras con valor de $17 \alpha \mathrm{OHP}$ en sangre mayor de $20 \mathrm{nmol} / \mathrm{L}$ se volvieron a analizar por duplicado en el mismo espécimen. Los resultados se interpretaron según la propuesta de Pearce y su grupo, que toma en cuenta el peso al nacimiento en 4 grupos diferentes menor o igual a $2,251 \mathrm{~g}$; de $1,751 \mathrm{a}$ 2,250 g; de 1,001 a 1,750 g; y menos de 1,000 g. ${ }^{19}$ También se estableció un valor de corte de emergencia (100 nmol/L) con el que todas las muestras se notificaron inmediatamente para que se efectuaran las pruebas confirmatorias y para la evaluación clínica del recién nacido. EI laboratorio que realizó el análisis de las muestras está inscrito en el Programa de Control de Calidad Externo de Calidad del Centers for Diseases Control and Prevention (CDC) de Atlanta y en el Programa de Evaluación Externa de la Calidad de la Fundación Bioquímica Argentina.

Estudios confirmatorios iniciales. Se efectuó la cuantificación sérica de la $17 \alpha \mathrm{OHP}$, aldosterona, androstenediona, cortisol, dehidroepiandrostenediona, testosterona total y la determinación de electrolitos séricos (cloro, sodio y potasio).

Diseño del estudio. Para el análisis del efecto de la edad del recién nacido al momento de la toma de la muestra y el peso al nacimiento en las concentraciones sanguíneas de $17 \alpha \mathrm{OHP}$, sólo se consideraron 1,236,822 muestras que tuvieron consignados estos datos en la ficha demográfica de identificación de la muestra, mismas que se dividieron en tres grupos según el momento de la toma de la muestra: Grupo 1 (G1), muestra 
de sangre para el tamiz obtenida antes de los 3 días de vida; Grupo 2 (G2), muestra obtenida entre el tercero y quinto días de vida; y Grupo 3 (G3), muestra obtenida después del quinto día de vida extrauterina. Estos grupos se subdividieron en cuatro grupos según el peso al momento del nacimiento (menos de 1,000 g; de 1,001 a 2,499 g; de 2,500 a 3,499 g y más de 3,500 g).

La prevalencia al nacimiento se calculó dividiendo el número de casos confirmados observados entre el número de recién nacidos tamizados. También se consignó el número de recién nacidos que, al localizar a los padres o tutores los refirieron como fallecidos. Por último, se calculó por entidad federativa la proporción de casos no localizados en relación con el número de recién nacidos sospechosos y la tasa de falla [no localizados (por todas las causas)/total de tamizados x 100,000 recién nacidos tamizados].

Los datos numéricos se presentan como promedios, medianas, percentiles, intervalos y porcentajes. El análisis estadístico se efectuó mediante el programa de acceso libre Comprehensive R Archive Network (https://cran.r-project. org). Se utilizó prueba de Mann-Whitney para comparar las diferencias entre los grupos. Se consideró significación estadística a los valores de $p<0.05$.

\section{RESULTADOS}

En las unidades médicas de la Secretaría de Salud se tamizaron 1,263,870 recién nacidos (Cuadro 1). Los casos sospechosos (rellamados), confirmados, no localizados, prevalencia al nacimiento y tasa de falla de localización se mencionan en el Cuadro 2. Las causas de falla en la localización de los casos sospechosos fueron variadas y algunas imputables a los padres (renuencia, migración o domicilio falso), al sistema de localización estatal, o se debieron al fallecimiento del recién nacido.
En la Figura 1 se observan claramente las diferencias en el número de rellamados obtenidos tomando un valor de corte fijo de $20 \mathrm{nmol} / \mathrm{L}$, comparado con el criterio de categorización por peso al nacimiento (5,960 muestras sospechosas, rellamado de $0.47 \%$ vs 1,806 , rellamado de $0.14 \%)$.

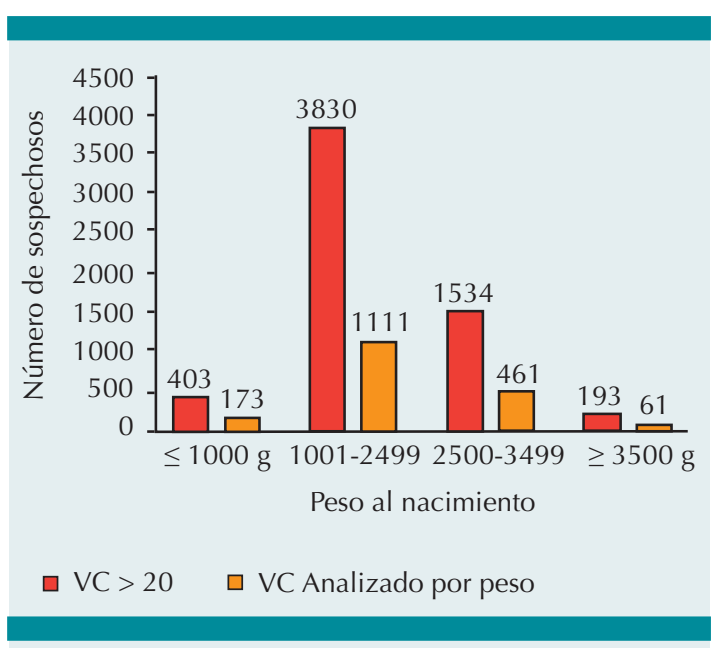

Figura 1. Comparación del número de muestras sospechosas solicitadas con el valor de corte (VC) fijo y tomando en consideración el peso del recién nacido. $n=1,806$ recién nacidos que tuvieron consignado el peso al nacimiento en la ficha de identificación.

Las concentraciones promedio de $17 \alpha \mathrm{OHP}$ en sangre en papel filtro de los recién nacidos prematuros fueron de $6.69 \mathrm{nmol} / \mathrm{L}$ y en los de término de $3.85 \mathrm{nmol} / \mathrm{L}$. En los recién nacidos sanos fueron de $3.88 \mathrm{nmol} / \mathrm{L}$ y en los enfermos se encontró $6.62 \mathrm{nmol} / \mathrm{L}$, con diferencias significativas en los grupos analizados. Figuras 2 A y B

El efecto de la edad del recién nacido al momento de la toma de la muestra y el peso al nacimiento en las concentraciones sanguíneas de $17 \alpha \mathrm{OHP}$, de acuerdo con los grupos y subgrupos establecidos, se observan en la Figura 3. Se encontraron diferencias significativas en los grupos: los valores de $17 \alpha \mathrm{OHP}$ fueron mayores en los recién nacidos con peso menor de 1,000 g (subgrupo 1) y disminuyen conforme el peso fue mayor. 


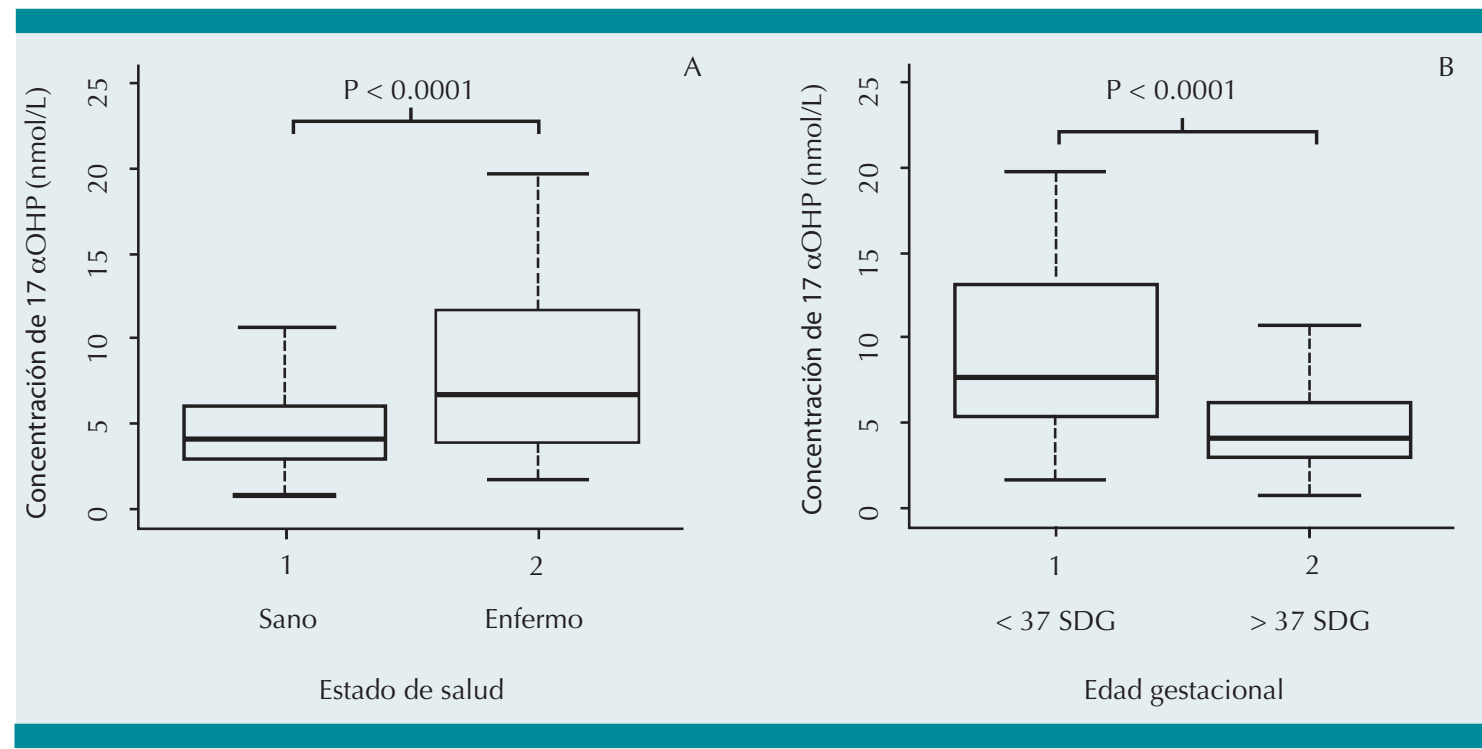

Figura 2. Concentraciones sanguíneas de $17 \alpha \mathrm{OHP}$ en recién nacidos mexicanos. A) Según su estado de salud, sanos $(1,179,625)$ y enfermos $(48,731)$. B) Según su edad gestacional, prematuros $(66,881)$ y de término $(1,173,005)$.

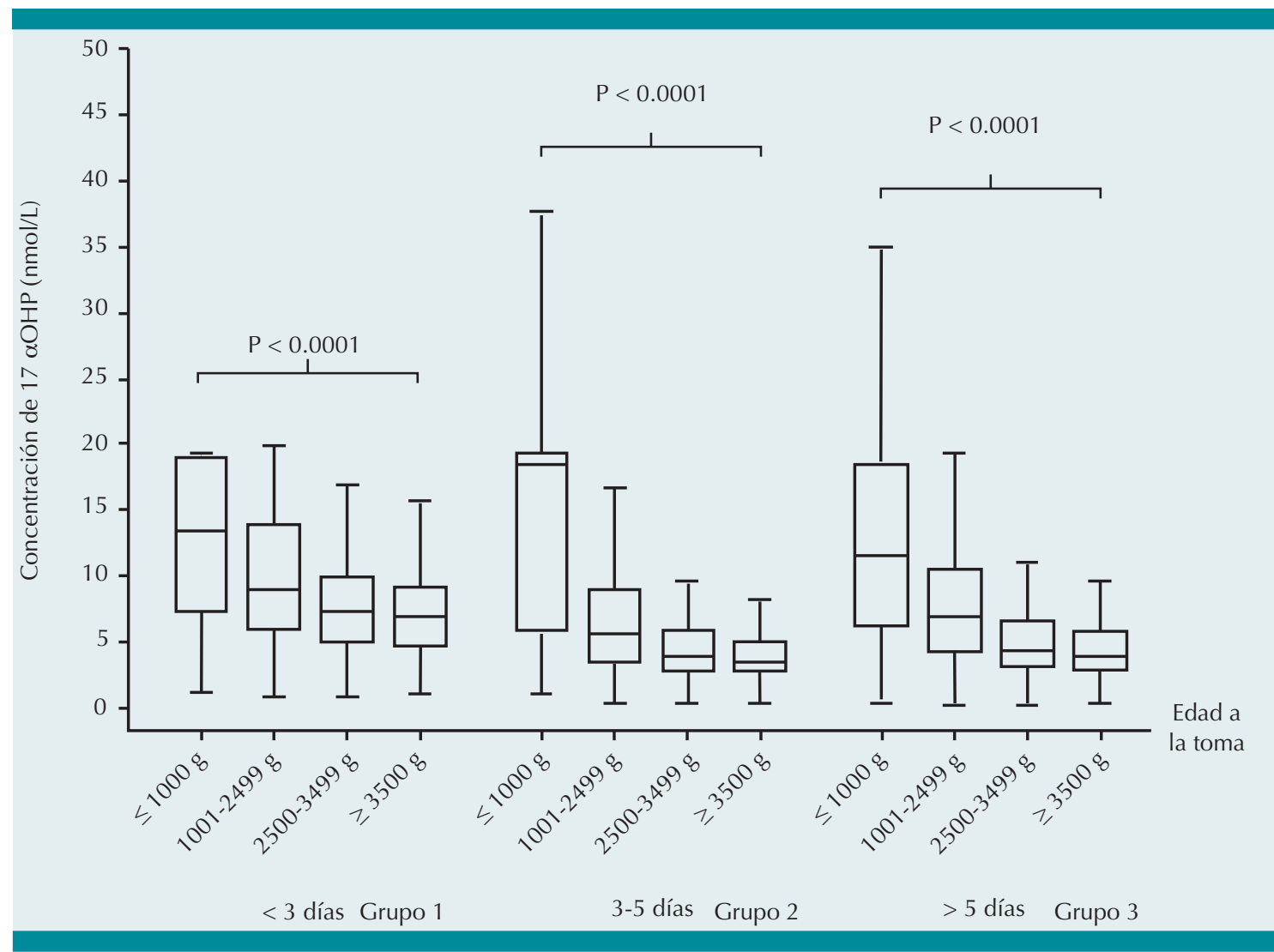

Figura 3. Concentraciones de $17 \alpha \mathrm{OHP}$ en sangre seca en papel filtro de los recién nacidos tamizados según su edad al momento de la toma de la muestra y peso al nacimiento. 
Cuadro 1. Recién nacidos tamizados, número de muestras adecuadas, edad de la toma de muestra de tamiz para hiperplasia suprarrenal congénita

\begin{tabular}{|c|c|c|c|c|c|c|c|c|}
\hline \multirow{3}{*}{$\begin{array}{l}\text { Entidad } \\
\text { Aguascalientes }\end{array}$} & \multirow{3}{*}{$\begin{array}{c}\text { RN } \\
\text { tamizados } \\
16,896\end{array}$} & \multirow{3}{*}{$\begin{array}{c}\begin{array}{c}\text { Muestras } \\
\text { adecuadas }\end{array} \\
16,878\end{array}$} & \multicolumn{6}{|c|}{ Edad de los RN al momento de toma de muestra } \\
\hline & & & \multicolumn{2}{|c|}{$<3$ días $\%$} & \multicolumn{2}{|c|}{3 - 5 días $\%$} & \multicolumn{2}{|c|}{$>5$ días $\%$} \\
\hline & & & 32 & 0.2 & 14,254 & 84.5 & 2,592 & 15.4 \\
\hline Baja California Norte & 24,341 & 24,290 & 45 & 0.2 & 19,582 & 80.6 & 4,663 & 19.2 \\
\hline Baja California Sur & 6,272 & 6,245 & 10 & 0.2 & 5,371 & 86 & 864 & 13.8 \\
\hline Campeche & 11,683 & 11,646 & 26 & 0.2 & 9,900 & 85 & 1,720 & 14.8 \\
\hline Chiapas & 59,709 & 59,204 & 273 & 0.5 & 38,233 & 64.6 & 20,698 & 35 \\
\hline Chuhuahua & 25,699 & 25,593 & 78 & 0.3 & 17,081 & 66.7 & 8,434 & 33 \\
\hline Ciudad de México & 42,795 & 42,694 & 108 & 0.3 & 35,769 & 83.8 & 6,817 & 16 \\
\hline Coahuila & 22,634 & 22,465 & 35 & 0.2 & 20,279 & 90.3 & 2,151 & 9.6 \\
\hline Colima & 7,657 & 7,647 & 231 & 3.0 & 6,454 & 84.4 & 962 & 12.6 \\
\hline Durango & 20,705 & 20,583 & 34 & 0.2 & 17,625 & 85.6 & 2,924 & 14.2 \\
\hline Estado de México & 150,948 & 150,558 & 204 & 0.1 & 147,274 & 97.8 & 3,080 & 2 \\
\hline Guanajuato & 101,602 & 100,935 & 320 & 0.3 & 82,110 & 81.3 & 18,505 & 18.3 \\
\hline Guerrero & 61,337 & 61,212 & 93 & 0.2 & 44,649 & 72.9 & 16,470 & 26.9 \\
\hline Hidalgo & 39,430 & 39,385 & 91 & 0.2 & 37,172 & 94.4 & 2,122 & 5.4 \\
\hline Jalisco & 77,353 & 77,051 & 141 & 0.2 & 56,162 & 72.9 & 20,748 & 26.9 \\
\hline Michoacán & 64,516 & 64,114 & 160 & 0.2 & 46,963 & 73.2 & 16,991 & 26.5 \\
\hline Morelos & 22,739 & 22,727 & 18 & 0.1 & 16,166 & 71.1 & 6,543 & 28.8 \\
\hline Nayarit & 9,981 & 9,940 & 45 & 0.5 & 9,404 & 94.6 & 491 & 4.9 \\
\hline Nuevo León & 31,997 & 31,928 & 24,260 & 76.0 & 5,125 & 16.1 & 2,543 & 8 \\
\hline Oaxaca & 48,151 & 47,848 & 176 & 0.4 & 44,187 & 92.3 & 3,485 & 7.3 \\
\hline Puebla & 96,041 & 95,773 & 143 & 0.1 & 67,103 & 70.1 & 28,527 & 29.8 \\
\hline Querétaro & 24,351 & 23,951 & 98 & 0.4 & 19,301 & 80.6 & 4,552 & 19 \\
\hline Quintana Roo & 19,930 & 19,772 & 48 & 0.2 & 17,992 & 91 & 1,732 & 8.8 \\
\hline San Luis Potosí & 28,759 & 28,644 & 116 & 0.4 & 22,654 & 79.1 & 5,874 & 20.5 \\
\hline Sinaloa & 26,928 & 26,908 & 36 & 0.1 & 20,433 & 75.9 & 6,439 & 23.9 \\
\hline Sonora & 21,318 & 21,273 & 38 & 0.2 & 12,560 & 59 & 8,675 & 40.8 \\
\hline Tabasco & 31,556 & 31,235 & 160 & 0.5 & 15,330 & 49.1 & 15,745 & 50.4 \\
\hline Tamaulipas & 35,588 & 35,484 & 120 & 0.3 & 31,629 & 89.1 & 3,735 & 10.5 \\
\hline Tlaxcala & 19,524 & 19,506 & 91 & 0.5 & 12,441 & 63.8 & 6,974 & 35.8 \\
\hline Veracruz & 80,306 & 78,739 & 171 & 0.2 & 66,857 & 84.9 & 11,711 & 14.9 \\
\hline Yucatán & 12,858 & 22,715 & 667 & 2.9 & 12,188 & 53.7 & 9,860 & 43.4 \\
\hline Zacatecas & 20,266 & 20,179 & 102 & 0.5 & 18,382 & 91.1 & 1,695 & 8.4 \\
\hline Total & $1,263,870$ & $1,267,122$ & 28,170 & 2.2 & 990,630 & 78.2 & 248,322 & 19.6 \\
\hline
\end{tabular}




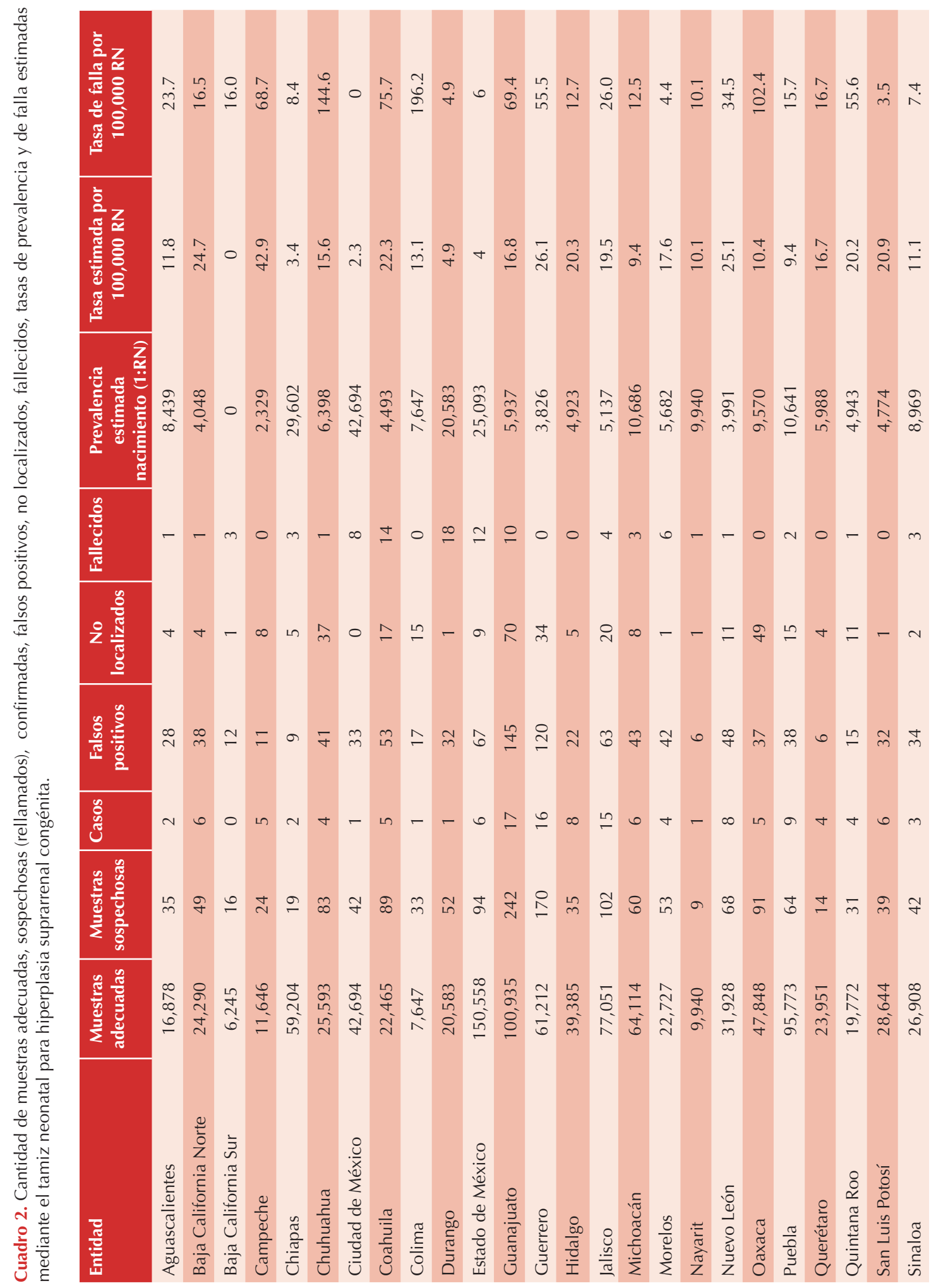




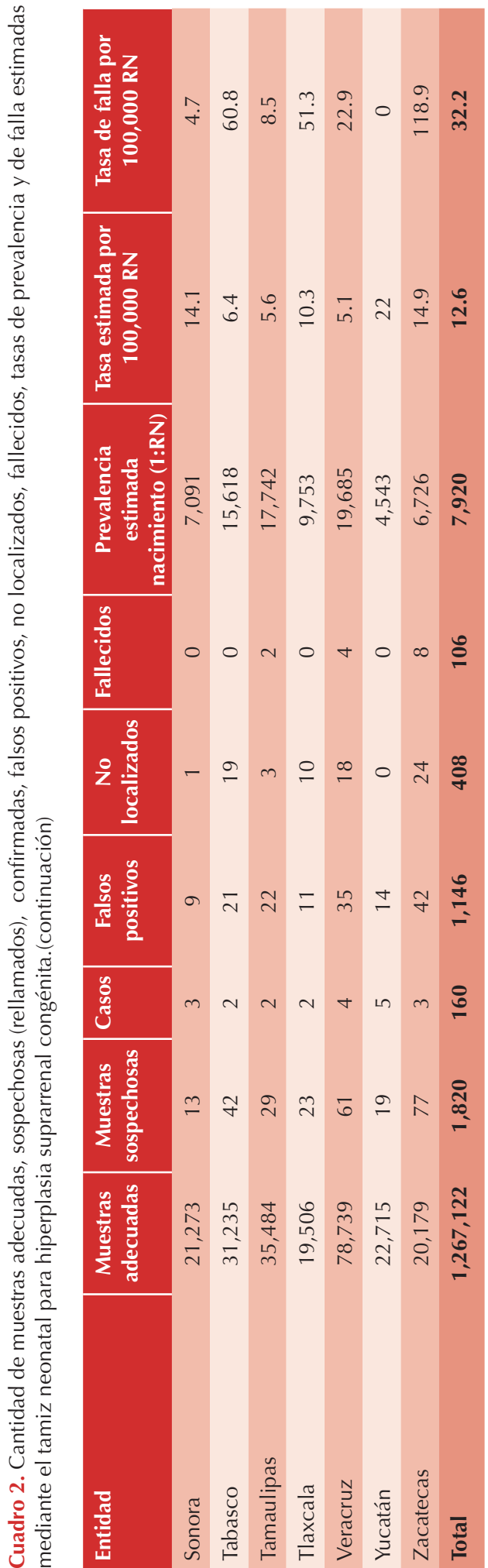

Los valores promedio de los estudios confirmatorios se encuentran en el Cuadro 3. De los casos confirmados, 9 tuvieron ambigüedad de genitales o sin dato, 45 fueron niñas y 101 niños.

Cuadro 3. Valores sanguíneos promedio de las pruebas confirmatorias

\begin{tabular}{|c|c|c|c|}
\hline Analito & Promedio & Máximo & Mínimo \\
\hline $\begin{array}{l}17 \text { hidroxiprogesterona } \\
\text { sangre en papel filtro }\end{array}$ & 207.87 & 1311.00 & 7.58 \\
\hline $\begin{array}{l}17 \text { hidroxiprogesterona } \\
\text { suero } \mathrm{ng} / \mathrm{mL}\end{array}$ & 41 & 311.92 & 4.65 \\
\hline $\begin{array}{l}\text { Aldosterona sérica } \\
\mathrm{pg} / \mathrm{mL}\end{array}$ & 2160.43 & 10389.00 & 401.08 \\
\hline Androstenediona $\mathrm{ng} / \mathrm{mL}$ & 16.21 & 102.00 & 0.33 \\
\hline Cortisol sérico $\mu \mathrm{g} / \mathrm{dL}$ & 8.13 & 49.60 & 1.06 \\
\hline $\begin{array}{l}\text { Dehidroepiandrosterona } \\
\mathrm{ng} / \mathrm{mL}\end{array}$ & 19.96 & 318.00 & 0.23 \\
\hline $\begin{array}{l}\text { Testosterona total } \\
\mathrm{nmol} / \mathrm{L}\end{array}$ & 14.49 & 249.50 & 0.78 \\
\hline $\mathrm{Cl}-($ Cloruro) $\mathrm{mmol} / \mathrm{L}$ & 98.99 & 116.00 & 72.00 \\
\hline $\mathrm{Cl}$-(Cloruro) mmol/L & 6 & 10.90 & 3.90 \\
\hline $\mathrm{Na}+$ (Sodio) $\mathrm{mmol} / \mathrm{L}$ & 133.42 & 146.00 & 112.00 \\
\hline
\end{tabular}

\section{DISCUSIÓN}

En este ensayo se exponen, detalladamente, las concentraciones sanguíneas de $17 \alpha \mathrm{OHP}$ obtenidas de más de un millón de recién nacidos tamizados en la Secretaría de Salud de México y el número de casos sospechosos de hiperplasia suprarrenal congénita confirmados y no localizados en el país.

Se sabe que la prematuridad, el estado de salud y el peso al nacimiento afectan las concentraciones sanguíneas de $17 \alpha \mathrm{OHP}$ neonatal. ${ }^{20,21,22}$ Los resultados de este ensayo muestran esta misma tendencia (Figuras 1 A y B) pues los recién nacidos de peso menor a $1000 \mathrm{~g}$ tuvieron los valores más altos de $17 \alpha \mathrm{OHP}$ en los distintos grupos de edad al momento de la toma de la muestra, pero también destaca que los valores del analito son significativamente mayores en las muestras tomadas antes del tercer día de vida, lo 
que contribuye al incremento de falsos positivos (Figura 2, Cuadro 3). La mayoría de las entidades federativas participantes toma las muestras entre el tercero y quinto días de vida extrauterina (78.2\%); destacan algunas diferencias: Nuevo León y Colima tuvieron una proporción mayor de toma de muestra temprana antes del tercer día de vida (76 y $3 \%$, respectivamente, Cuadro 1 ).

La prevalencia al nacimiento de hiperplasia suprarrenal congénita obtenida en esta investigación fue de 12.6 por cada 100,000 recién nacidos tamizados; es decir, 1:7920 recién nacidos tamizados. Estos datos son similares a las cifras encontradas por el IMSS. ${ }^{7}$ Los estados con mayor prevalencia fueron: Campeche, Guerrero y Nuevo León con 42.9, 26.1 y 25.1 x 100,000 recién nacidos tamizados, respectivamente. En todos los estados de la República se encontraron casos de hiperplasia suprarrenal congénita, excepto en Baja California Sur, pero en este estado la tasa de falla de localización fue elevada (16.0 x 10, 000 recién nacidos). Cuadro 2

En algunos países industrializados, con programas muy bien establecidos de tamiz para hiperplasia suprarrenal congénita, se describe una proporción de falsos positivos menor de $0.5 \%$, pero hay programas que reportan entre 0.02 a $0.25 \% .^{23}$ En este ensayo se encontraron $0.09 \%$ de falsos positivos, valor subestimado puesto que una proporción significativa de sospechosos no pudieron localizarse y, por lo tanto, concluir el proceso del tamiz neonatal. El costo económico y psicosocial asociado con los falsos positivos es grande e incluye desde el análisis por duplicado de las muestras por arriba del valor de corte, notificación del caso sospechoso, localización y confirmación, si además se le agrega el gasto del seguimiento clínico, el costo se vuelve significativamente mayor. ${ }^{12}$

Una aportación de esta investigación es demostrar en la población de recién nacidos mexicanos la utilidad de la interpretación del resultado mediante el criterio del peso al nacimiento, ${ }^{17}$ puesto que cuando no se hace ese ajuste, el número de rellamados es 3.3 veces más alto $(0.47 \%)$ comparado con el ajustado (0.14\%). Figura 3

Un hallazgo sorprendente de este ensayo es el elevado número recién nacidos fallecidos del grupo de casos sospechosos (106 muertos por cada 1820 sospechosos, 5.82\%) con un máximo en Durango (18 muertos por cada 52 sospechosos, $34.6 \%$ ) y en la Ciudad de México (8 muertos por cada 42 sospechosos, 19.04\%) (Cuadro 2). Entre esos recién nacidos fallecidos existe la posibilidad de que haya un número de casos verdaderos de la enfermedad que no pudieron confirmarse y tratarse a tiempo, a pesar de haber sido tamizados. El advenimiento del tamiz neonatal para la detección de hiperplasia suprarrenal congénita representa uno de los programas más exitosos para disminuir la mortalidad en el periodo neonatal tardío, ${ }^{2}$ y el tamiz para esta enfermedad cumple con los criterios clásicos de Wilson y Jungner. ${ }^{24}$ Sin embargo, se requiere robustecer los sistemas postanalíticos y de seguimiento de tamiz neonatal para que todo sospechoso de la enfermedad sea localizado, confirmado y reciba atención médica inmediata.

Una limitante de este estudio fue que, para la confirmación del diagnóstico, solo se realizó un perfil bioquímico general de esteroides y electrolitos, y actualmente se sabe que pueden efectuarse estudios más completos que incluyan la espectrometría de masas en tándem, pruebas farmacológicas especializadas de estimulación de la hormona adrenocorticotrópica con cosyntropin, y pruebas genéticas para confirmar el diagnóstico. ${ }^{2,25}$

\section{CONCLUSIÓN}

La prevalencia al nacimiento de hiperplasia suprarrenal congénita en este trabajo fue de 
12.6 por cada 100,000 (1:7920) recién nacidos tamizados en la Secretaría de Salud de México. Para disminuir el número de casos sospechosos no localizados es necesario reforzar el componente postanalítico del tamiz neonatal.

\section{REFERENCIAS}

1. Delle Piane L, Rinaudo PF, Miller WL. 150 years of congenital adrenal hyperplasia: translation and commentary of De Crecchio's classic paper from 1865. Endocrinology. 2015; 156 (4):1210-17.

2. El-Maouche D, Arlt W, Merke DP. Congenital adrenal hyperplasia. Lancet. 2017; 390(10108):2194-210.

3. Antal Z, Zhou P. Congenital Adrenal Hyperplasia. Pediatr Rev. 2009; 30(7): e49-57.

4. Manoli I, Kanaka-Gantenbein C, Voutetakis A, Maniati-Christidi M, Dacou-Voutetakis C. Early growth, pubertal development, body mass index and final height of patients with congenital adrenal hyperplasia: factors influencing the outcome. Clin Endocrinol (Oxford) 2002; 57(5): 669-676.

5. Speiser PW, Azziz R, Baskin LS, Ghizzoni L, Hensle TW, Merke $D P$, et al. Congenital adrenal hyperplasia due to steroid 21-hydroxylase deficiency: an Endocrine Society clinical practice guideline. J Clin Endocrinol Metab. 2010; 95(9):4133-60.

6. Paz-Valiñas L, Varela-Lema L, Atienza-Merino G. Cribado neonatal de la hiperplasia suprarrenal congénita. Revisión sistemática, Red Española de Agencias de Evaluación de Tecnologías y Prestaciones del Sistema Nacional de Salud. Axencia de Avaliación de Tecnoloxias Sanitarias de Galicia. 2014. Informes de Evaluación de Tecnologías Sanitarias. Galicia, 2014. 139 pp.

7. CENETEC. Guía de Práctica Clínica, Tamizaje, Diagnóstico y Tratamiento del Paciente con Hiperplasia Suprarrenal Congénita por Deficiencia de 21 Hidroxilasa (IMSS-715-114). México: CENETEC; 2014. Disponible en: http://www.cenetec.salud.gob.mx/descargas/gpc/CatalogoMaes.

8. Marumudi E, Khadgawat R, Surana V, Shabir I, Joseph A, Ariachery $C$, Ammini. Diagnosis and management of classical congenital adrenal hiperplasia. Steroids. 2013; 78 (8): 741-6.

9. Thil'en A, Nordenström A, Hagenfedt L, von Döbelm $U$, Guthenberg C, Larsson A. Benefits of neonatal screening for congenital adrenal hyperplasia 21-hydroxylase deficiency in Sweden. Pediatrics. 1998; 101(4):E11.

10. Grosse SD, Van Vliet G. How many deaths can be prevented by newborn screening for congenital adrenal hyperplasia? Horm Res. 2007;67(6):284-91.

11. Heather NL, Seneviratne SN, Webster D, Derraik JG, Jefferies C, Carll J, et al. Newborn screening for congenital adrenal hyperplasia in New Zealand, 1994-2013.J Clin Endocrinol Metab. 2015; 100(3):1002-8.

12. Olgemöller B, Roscher AA, Liebl B, Fingerhut R.Screening for congenital adrenal hyperplasia: adjustment of 17-hydroxyprogesterone cut-off values to both age and birth weight markedly improves the predictive value. J Clin Endocrinol Metab. 2003; 88(12):5790-4.

13. Wong $\mathrm{T}$, Shackleton $\mathrm{CH}$, Covey TR, Ellis G.Identification of the steroids in neonatal plasma that interfere with 17alpha-hydroxyprogesterone radioimmunoassays. Clin Chem. 1992;38(9):1830-7.

14. Sarafoglou K, Banks K, Kyllo J, Pittock S, Thomas W. Cases of congenital adrenal hyperplasia missed by newborn screening in Minnesota. JAMA. 2012; 307(22):2371-4. doi: 10.1001/ jama.2012.5281.

15. Coulm B, Coste J, Tardy V, Ecosse E, Roussey M, DHCSF Study Group. Efficiency of neonatal screening for congenital adrenal hyperplasia due to 21-hydroxylase deficiency in children born in mainland France between 1996 and 2003. Arch Pediatr Adolesc Med. 2012; 166(2):113-20. doi: 10.1001/ archpediatrics.2011.774.

16. Secretaría de Salud, Diario Oficial de la Federación. Ley General de Salud, Artículo 61, Fracción II, reforma del 25/01/2013.

17. Norma Oficial Mexicana NOM-034-SSA2-2013, Para la prevención y control de los defectos al nacimiento. Diario Oficial de la Federación, 24/06/2014, México.

18. Norma Oficial Mexicana NOM-007-SSA2-2016, Para la atención de la mujer durante el embarazo, parto y puerperio, y de la persona recién nacida. Diario Oficial de la Federación, 01/04/2016, México.

19. Pearce M, Dauerer E, DiRienzo AG, Caggana M, Tavakoli NP. The influence of seasonality and manufacturer kit lot changes on 17 $\alpha$-hydroxyprogesterone measurements and referral rates of congenital adrenal hyperplasia in newborns. Eur J Pediatr. 2017; 176(1):121-9.

20. Gruñeiro-Papendieck L, Prieto L, Chiesa A, Bengolea S, Bossi $G$, Bergadá C. Neonatal screening program for congenital adrenal hyperplasia: adjustments to the recall protocol. Horm Res. 2001;55(6):271-7.

21. Bornstein S, Allolio B, Arlt W, Barthel A, Don-Wauchope A, Hammer G, Husebye E, Merke D, Murad M, Stratakis C, Torpy D. Diagnosis and Treatment of Primary Adrenal Insufficiency: An Endocrine Society Clinical Practice Guideline. J Clin Endocrinol Metab. 2016; 101 (2): 364-389.

22. Bonfig W, Bechtold S, Schmidt H, Knorr D, Schwarz H. Reduced Final Height Outcome in Congenital Adrenal Hyperplasia under Prednisone Treatment: Deceleration of Growth Velocity during Puberty. J Clin Endocrinol Metab. 2015; 92 (5): 1635-9.

23. Wilson JMG, Jungner G. Principles of Screening for Disease. Geneva: World Health Organization, 1968.

24. Tsuji A, Konishi K, Hasegawa S, Anazawa A, Onishi T, Ono M, et al. Newborn screening for congenital adrenal hyperplasia in Tokyo, Japan from 1989 to 2013: a retrospective population-based study. BMC Pediatr. 2015; 15:209. DOI 10.1186/ s12887-015-0529-y.

25. Struja T, Briner L, Meier A, Kutz A, and Mundwiler E, Huber $A$, et al. Diagnostic accuracy of basal cortisol level to predict adrenal insufficiency in cosyntropin testing: results from an observational cohort study with 804 patients. Endocr Pract. 2017; 23(8):949-61. 